\title{
L'université d'Harvard est-elle trop souvent citée en exemple?
}

Monsieur,

Le fait est que l'université d'Harvard est souvent prise comme exemple à suivre ${ }^{[1]}$. Rien de surprenant à cela. Elle a une excellente réputation depuis plusieurs siècles. Elle produit des chercheurs qui ne se contentent pas de chercher, mais qui trouvent souvent et récoltent les Prix Nobel.

Dans la foulée les admirateurs en arrivent à croire aussi à la haute qualité de son enseignement en postulant que, si les résultats sont bons c'est parce que les professeurs d'Harvard savent bien enseigner.

Ma persistante théorie est que cette relation de causalité n'existe pas plus là que dans n'importe quelle autre université à travers le monde. J'ai même le mauvais goût de penser que ces bons résultats sont obtenus malgré le processus pédagogique ambiant et non pas grâce à lui.

Un article du New-York Times ${ }^{[2]}$ apporte de l'eau à mon moulin.

Éric Mazur, 52 ans, a étudié la physique à l'université de Leiden en Hollande. Il est devenu «Gordon McKay Professor of Applied Physics » à Harvard en 1980. Que dit-il ? Il reproche au processus d'enseignement d'être trop centré sur les connaissances factuelles et l'apprentissage par cœur (focused on facts and rote memorization) au lieu de l'être sur l'acquisition d'un raisonnement scientifique. À Leyden il se souvient que «les conférences magistrales étaient mortelles et frustrantes. Aux États-Unis aussi, dans les amphis, les étudiants se concentrent sur la mémorisation brute de données factuelles. Ceux qui obtiennent les meilleures notes aux examens sont ceux qui sont les plus capables de régurgiter de l'information ». [...] «Quand j'ai commencé à enseigner à Harvard dans les années 80, je ne me posais pas de questions d'ordre pédagogique. J'ai fait des conférences magistrales comme tout le monde. Et le feedback fut positif. Mes étudiants obtinrent de bons résultats à des examens que je considérais comme étant difficiles. Mais lorsque j'ai construit des épreuves mesurant la compréhension de concepts scientifiques, environ la moitié d'entre eux montrèrent qu'ils n'avaient aucune idée de ce que pouvait être la mécanique newtonienne. [...] Jusqu'alors je faisais de la science devant eux au lieu de les aider à le faire eux-mêmes. Si j'avais été prof de piano me serais-je limité à leur dire «asseyez vous, je vais vous jouer du piano »? ». [...] « À Harvard chaque enseignant est libre d'enseigner comme bon lui semble. Il n'y a pas beaucoup de pollinisation croisée. Maintenant au moins, pour mes étudiants l'étude individuelle du contenu théorique doit se faire avant le cours lui-même. Pendant le cours la discussion entre les étudiants porte sur des applications dans la vie de tous les jours et, à l'occasion de travaux en petits groupes, ils utilisent des concepts scientifiques pour résoudre ensemble un problème. Les examens sont «à livre ouvert », comme dans la vie professionnelle réelle».

Même la très scientifique revue Science ${ }^{[3]}$ se réjouit que «la France prenne son inspiration auprès de la Harvard School of Public Health». La question reste de savoir si on va continuer ou non de faire des conférences magistrales.

Jean-Jacques GUILBERT

5 avenue du Mail 1205 Genève, Suisse Mailto : guilbertjj@yahoo.fr 


\section{Références}

1. Flahault A. L'École des Hautes Études en Santé Publique (EHESP) : I. Quel projet pour la France ? Bull Acad Nat Med 2007;191:325-37.

2. Dreifus C. A Conversation With Eric Mazur. Using the «Beauties of Physics» to Conquer Science Illiteracy.
New York Times 2007, July 17. [On-line] Disponible sur : http://www . nytimes.com/2007/07/17/science/ 17conv.html?_r=1\&scp=2\&sq=Mazur\%20E\&st=cse \& oref=slogin

3. Enserink M. France Launches Public Health School à l'Anglo-Saxonne. Science 2008 ;319:397 